\title{
Genome editing: A breakthrough in life science and medicine
}

\author{
Izuho Hatada and Takuro Horii \\ Laboratory of Genome Science, Biosignal Genome Resource Center, Institute for Molecular and Cellular Regulation, Gunma \\ University, Maebashi 371-8512, Japan
}

\begin{abstract}
Genome editing technologies represent a major breakthrough that has dramatically altered strategies in a wide range of biological studies. Genome editing simplifies and accelerates the creation of animal disease models and enables construction of models in most animal species, even those that are not amenable to conventional gene targeting technology.
\end{abstract}

Key words: Genome editing, CRISPR/Cas9, Cas9, ZFN, TALEN

FOR MANY YEARS, conventional gene targeting was widely used to generate knockout mice. However, the conventional method exploits spontaneous homologous recombination, which is a rare event. Therefore, this technology was not efficient enough to construct gene-targeted mice directly from fertilized eggs, which are available in limited numbers for experiments. To compensate for this shortcoming, researchers using this technique must obtain a large number of germline-competent cells and apply selection methods. Consequently, embryonic stem cells (ESCs) are used instead of eggs for conventional gene targeting because these cells can be relatively easily propagated and subjected to drug selection. However, generation of germline-competent ESCs is extremely difficult, as reflected by the fact that development of ESCs in rats took 20 years after the first production of mouse ESCs. Moreover, it has become clear that the phenotypes of mouse knockouts of disease-associated genes do not always accurately reflect human disease phenotypes. Furthermore, mice cannot be used for studies of higher-order brain functions. Therefore, for both preclinical testing and fundamental research, it is important to construct disease models using large animals such as pig and monkey. However, it is currently

Submitted Dec. 16, 2015; Accepted Dec. 16, 2015 as EJ15-0716 Released online in J-STAGE as advance publication Dec. 22, 2015 Correspondence to: Izuho Hatada, Laboratory of Genome Science, Biosignal Genome Resource Center, Institute for Molecular and Cellular Regulation, Gunma University, 3-39-15 Showa-machi, Maebashi 371-8512, Japan. E-mail: hatada@gunma-u.ac.jp impossible to use conventional gene targeting methods to generate disease models in animals for which ESCs are not available.

To overcome these challenges, technological innovation was required. Recent advances in artificial restriction enzymes, which enable specific cleavage, have provided one such solution. In this approach, specific cleavage of the target locus is followed by incidental deletion by endogenous nucleases, which can be repaired by spontaneous non-homologous end joining (NHEJ) or homology-directed repair (HDR). NHEJ results in a small deletion or insertion at the target locus (Fig. 1), whereas HDR can yield a knock-in at the target locus if a donor sequence is provided. These artificial restriction enzymes, collectively termed genome editing technologies, are represented by three main categories: zinc-finger nucleases (ZFNs) [1], transcription activator-like effector nucleases (TALENs [2], and the clustered regularly interspaced short palindromic repeat (CRISPR)/Cas9 system [3]. Using these systems, it is possible to efficiently produce gene-targeted animals directly from eggs. In addition, even biallelic mutants can be produced by gene editing, dramatically shortening the time required to generate gene-targeted mice. It takes more than a year to obtain homozygous mutant mice using the conventional gene targeting method based on ESCs, whereas genome editing technologies can produce biallelic mutant mice within 1 month, especially when using the CRISPR/Cas9 method.

ZFNs and TALENs comprise customized DNA- 


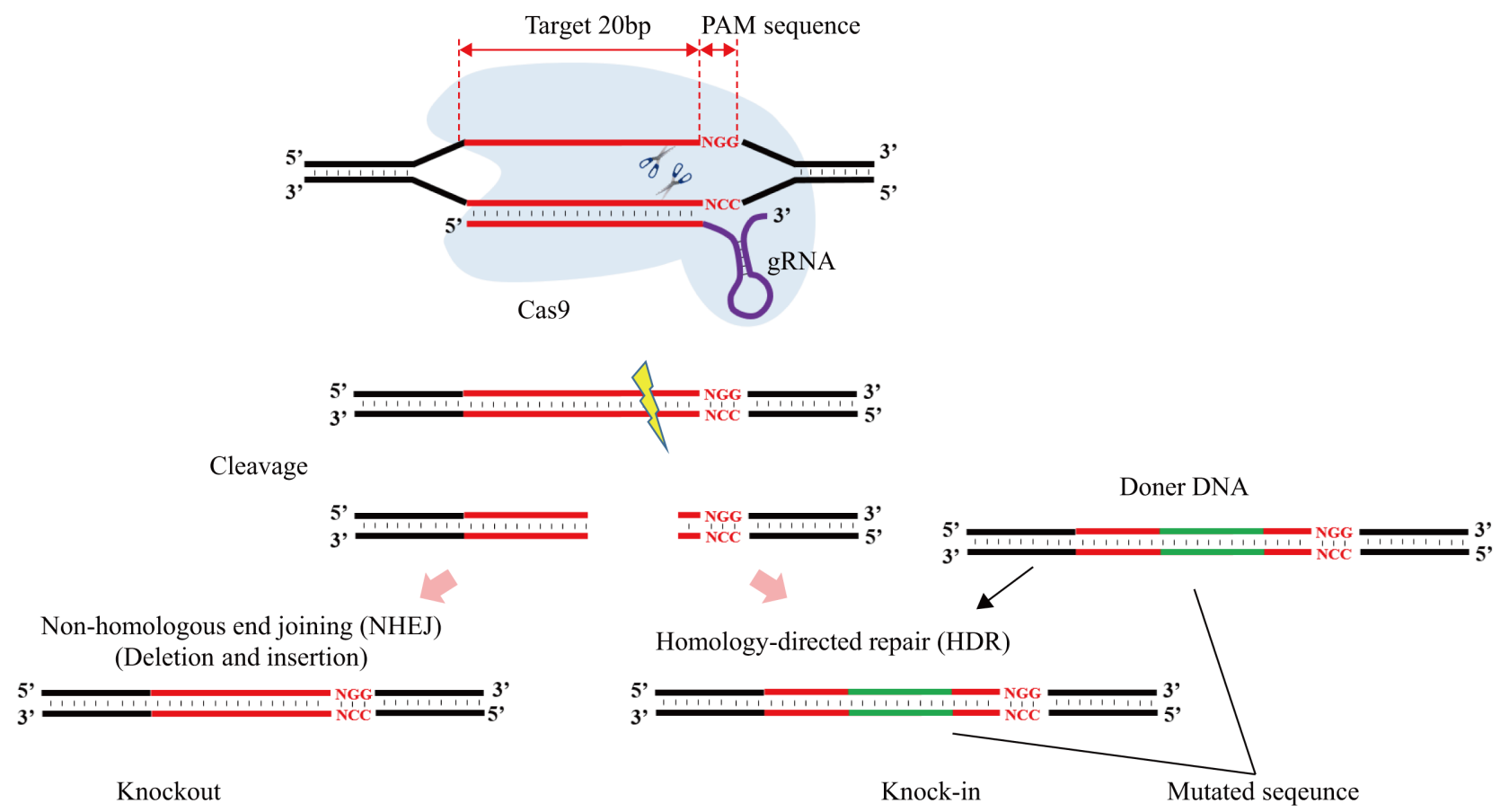

Fig. 1 Schematic of the CRISPR/Cas9 system. The Cas9 endonuclease and gRNA complex cleave the target sequence. Cleavage requires the PAM (NGG) adjacent to the $3^{\prime}$ end of the 20-bp target sequence. Cleavage of the target is followed by either spontaneous non-homologous end joining (NHEJ) or homology-directed repair (HDR). NHEJ results in a small deletion or insertion at the targeted locus, whereas HDR can generate a knock-in at the targeted locus if a donor sequence is supplied. Cas9, CRISPR associated protein 9; PAM, protospacer adjacent motif.

binding domains that recognize target sequences and cleavage domains derived from the restriction enzyme FokI. Zinc-finger domains harbor several amino acids on the surface of the $\alpha$-helix that can selectively contact three base pairs within the DNA strand. In ZFNs, artificial arrays of multiple zinc-finger domains enable specific recognition of long DNA sequences. Transcription activator-like effectors (TALEs) are naturally occurring DNA-binding proteins from bacteria that contain arrays of multiple 34-amino acid units. Each unit specifically recognizes a single base, and artificial arrays of these units can specifically bind to longer DNA sequences.

\section{History of CRISPR}

As Marcus Tullius Cicero said, "Omnium rerum principia parva sunt (the beginnings of all things are small)." Without small findings, great achievements are impossible. In 1987, a young Japanese scientist, Yoshizumi Ishino, described short palindromic repeat sequences in the downstream region of the iap gene of E. coli [4]. These sequences are now known as CRISPRs (Clustered Regularly Interspaced Short Palindromic Repeats). Ishino's paper concludes: "So far, no sequences homologous to these have been found elsewhere in procaryotes, and the biological significance of these sequences is not known." At that time, the tools necessary for further investigate did not yet exist. The next advance required to solve this mystery was not obtained until 2000, in the era of the genome projects, when the Spanish scientist Francisco Mojica performed a comparative analysis of prokaryote genomes revealing that CRISPRs are common in multiple species [5].

In 2005, three teams of scientists independently reported that short spacer sequences between CRISPRs are homologous to fragments of viral (bacteriophage) genomes [6-8]. In 2006, Eugene Koonin proposed that CRISPRs are a defense mechanism that provide immunological memory [9]. In 2007, his hypothesis was verified by Rodolphe Barrangou, a microbiologist working at the yogurt manufacturer Danisco. Protection of cultures against viral infections is a critical issue for yogurt producers. Barrangou's team found that a lactic acid bacterium lacking the spacer 
sequences between CRISPR repeats lost its resistance to viruses [10]. These findings suggested that bacteria have an adaptive immune system that incorporates short fragments of foreign (in this case, viral) DNA as spacers within CRISPR loci. Subsequently, several groups confirmed the existence of adaptive immunity in bacteria and elucidated the underlying mechanisms (Fig. 2). The CRISPR cluster is expressed as the precrRNA (CRISPR RNA), which is processed by a transactivating CRISPR RNA (tracrRNA) complementary to a part of pre-crRNA and matured into crRNA.

On August 17, 2012, Emmanuelle Charpentier, Jennifer Doudna, and their colleagues published an exciting collaborative study, "A Programmable DualRNA-guided DNA Endonuclease in Adaptive Bacterial Immunity," in the journal Science [3]. CrRNA consists of two parts: a sequence complementary to the tracrRNA and a 20-bp sequence complementary to the viral target sequence. They found that crRNA that is base-paired to trans-activating crRNA (tracrRNA) forms a two-RNA structure that directs Cas9 (CRISPRassociated protein 9) to introduce specific doublestranded breaks in target DNA. Site-specific cleavage requires both base-pairing complementarity between the crRNA and the target DNA and a short sequence called the protospacer adjacent motif (PAM) next to the complementary region in the target DNA. Finally, they showed that only two components, Cas9 protein and a tracrRNA-crRNA chimera called guide RNA (gRNA), are required for sequence-specific cleavage of DNA in vitro (Fig. 1). Many readers of that paper predicted that if RNA-guided nucleases could be adapted to work in mammalian cells or fertilized eggs, knockout or knockout animals could be easily produced. Indeed, 5 months after the original paper was published, four studies demonstrated that the CRISPR/Cas9 system can be used to produce knockouts in mammalian cells [11-14]. Fours month after that, another group reported that knockout mice can be easily generated by direct injection of CRISPR/Cas9 into oocytes [15].

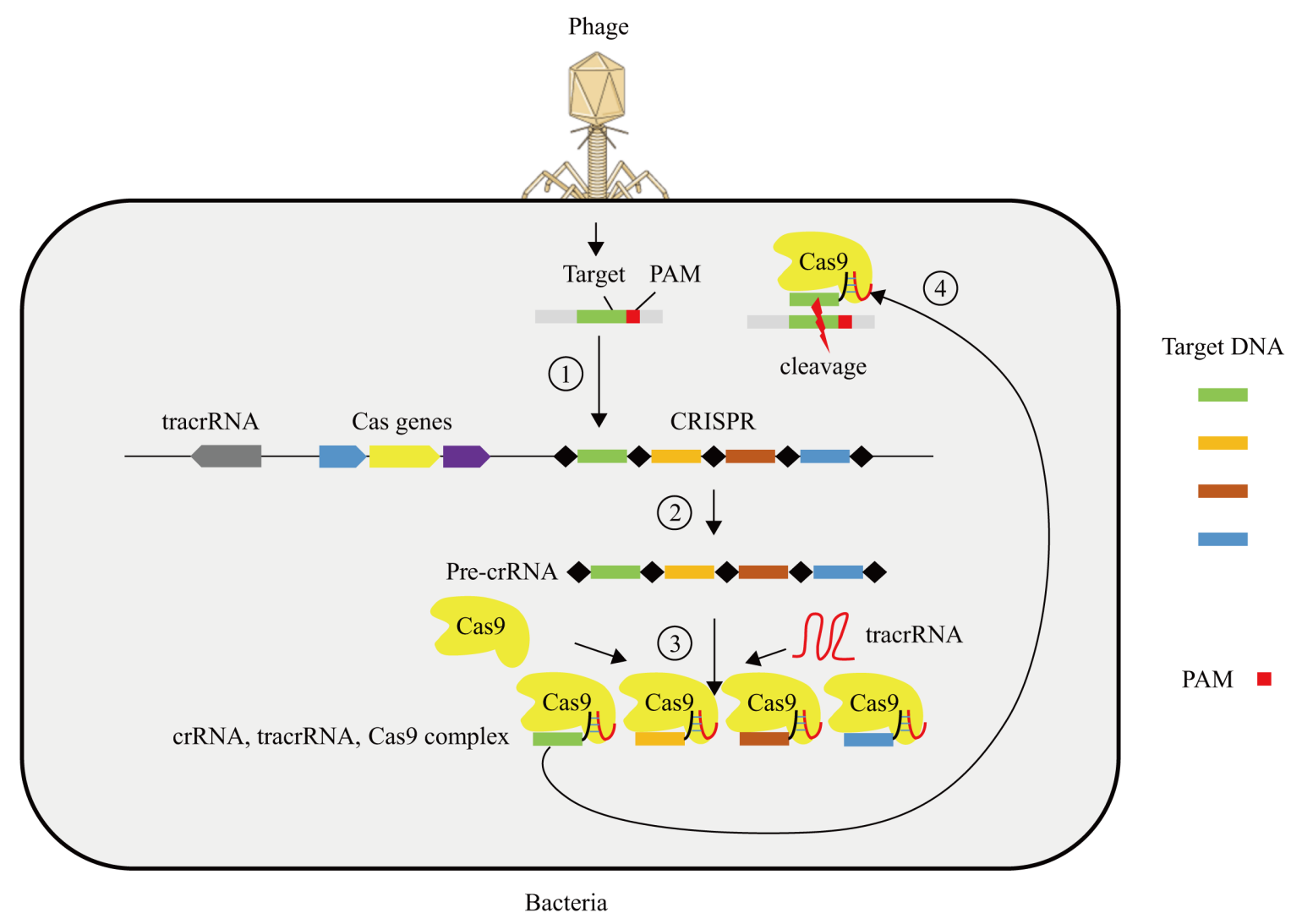

Fig. 2 CRISPR/Cas9 is an adaptive bacterial immunity system comprising four steps. (1) Target bacteriophage DNA located upstream of the PAM is cut out and integrated into the CRISPR locus; (2) The CRISPR locus is transcribed as pre-crRNA; (3) The crRNA matures and forms a complex with tracrRNA and Cas9; (4) The protein-RNA cleaves the target upstream of the PAM. 


\section{Application of CRISPR/Cas9}

Following the advent of highly efficient, simple, and straightforward tools such as CRISPR/Cas9, genome editing opened the door to manipulating the genomes of a wide range of species (Fig. 3). For example, genome editing in most mammals can be performed by direct injection of Cas9 and gRNA into fertilized eggs. To date, this approach has been used to generate genome-edited animals in rat, pig, monkey, rabbit, zebra fish, Drosophila, C. elegans, and other species. CRISPR/Cas9 can be also used for gene targeting in cultured cells. Before the development of genome editing technologies, it was very difficult to manipulate the genomes of cultured cells, with the exception of special cell types such as ESCs. Therefore, most researchers elected to produce mouse embryonic fibroblast cells from gene-targeted mice, and then use these cells for experiments. With CRISPR/Cas9, however, gene targeting can be performed in almost all cultured cells, dramatically shortening the time required for experiments from more than 1 year to 1 month. Pluripotent stem cells, including induced pluripotent stem cells (iPSCs), can be also manipulated by CRISPR/Cas9 gene targeting [16, 17] (Fig. 3). CRISPR/Cas9 is an efficient method for both constructing disease models in wild-type iPSCs [16] and for correcting disease genes in patient-derived iPSCs [18].

Simultaneous targeting of multiple genes can be achieved using CRISPR/Cas9 technology [15] (Fig. 3 ). This has enabled, for example, the creation of liver tumors by direct injection of CRISPR/Cas9 to delete
Pten and p53 in the same cells [19]. This method has been used to disrupt as many as 62 endogenous retrovirus loci, which pose potential risks in clinical application, in order to generate pigs that are safe to use in pig-to-human xenotransplantation [20].

Many groups are trying to apply this technology to gene therapy; to this end, several model experiments have been performed (Fig. 3). In mice, CRISPR/Cas9 has been used to perform germline correction of multiple genetic diseases, including cataract and muscular dystrophy [21, 22], and the technology was also used to make corrections in somatic cells. For example, in a mouse model of the human disease hereditary tyrosinemia, the CRISPR/Cas9 system was used to correct the underlying Fah mutation in hepatocytes. Hydrodynamic injection resulted in initial expression of the wild-type Fah protein in only 1/250 liver cells; however, the corrected cells had a growth advantage, and in the absence of therapeutic medication they proliferated to constitute $34 \%$ of the hepatocyte population after 1 month [23]. Infectious diseases are also promising targets for genome editing gene therapy; disruption of both viral genes and virus receptors is an attractive approach for such therapies. For example, human hematopoietic stem/progenitor cells have been modified by ZFNs targeting the HIV co-receptor, CCR5, to control HIV-1 in vivo [24]. This therapy, developed by Sangamo BioSciences, is now in phase II clinical trials.

New technologies have been developed by combining CRISPR/Cas9 with other techniques (Fig. 3). A Cas9 mutant defective for nuclease activity (dCas9) is frequently used for these purposes. Because the

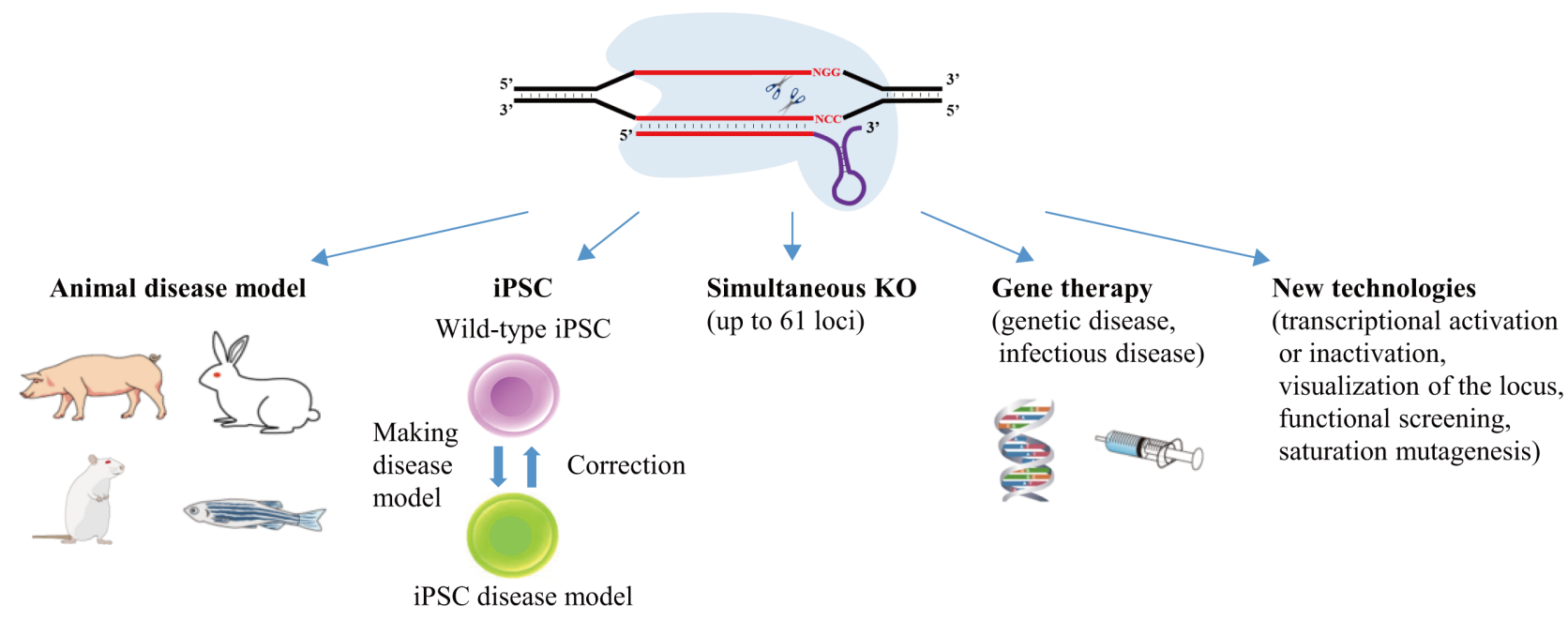

Fig. 3 Various applications of CRISPR/Cas9 
mutant still retains gRNA-dependent DNA-binding activity, it can recruit multiple enzymatic activities to specific loci. For example, a fusion protein of dCas9 and a transactivation domain such as VP64 can be used to activate gene promoters in a sequence-specific manner [25]. Inversely, fusion with an inactivation domain can be used to inactivate promoters [26]. Fusion with EGFP enables visualization of specific loci [27], and fusion with a tag protein enables chromatin immunoprecipitation of specific loci [28]. In combination with parallel on-chip gene synthesis, gRNA libraries for whole genes have been generated and used for functional screening [29], including screens for cancer-related genes [30]. Another example is the use of CRISPR/Cas9 for saturation mutagenesis, which is the generation of all possible mutations at a specific site or within narrow region of a gene; this is a powerful tool for both functional analysis of genes and directed evolution of functional enzymes. Saturation mutagenesis of an exon of an endogenous gene can be achieved by CRISPR/Cas9-guided cleavage of specific loci and multiplex homology-directed repair using a complex library of donor templates [31].

\section{Future Prospects}

In the near future, genome editing must progress in three major directions. The first is improvement of CRISPR/Cas9 and development of superior alternatives. One shortcoming of the current system is that it sometimes exerts off-target effects, although this occurs primarily in cancer cells and does not frequently happen in genome-edited mice. This problem has been solved using modifications to the CRISPR/ Cas9 system, such as the double-nicking method [32]. The requirement for the PAM, which limits the choice of sites to be targeted, is another important concern.
The PAM of SpCas9 is NGG; if this could be changed to a shorter sequence or simply to another specific sequence, the resultant modified system could be used to edit a broader range of genomic targets. Along these lines, engineered CRISPR/Cas9 nucleases with altered PAM specificities [33], and another CRISPR system using Cpf1 nuclease [34] with a T-rich PAM, were reported in recent studies.

The second important challenge is development of new technologies such as those described in the previous chapter. Many new combination technologies have been reported in a variety of fields, and these tools will merit close attention in the years to come.

The third aspect is application to biomedicine, including gene therapy. In this context, safety must be guaranteed and delivery improved. With regard to safety, genome-edited cells can be tested for chromosomal rearrangement, duplication, or loss using copy number variation arrays, and high-throughput sequencing of whole genomes can be used to identify clones with minimal mutation loads. By contrast, improving delivery will be much more challenging. In general, delivery of nucleic acids into cells is accomplished using viral vectors, whose insert size is limited. The size of the most frequently used Cas 9 gene from Streptococcus pyogenes (SpCas9) is 4.2 kilobases (kb), too long for most viral vectors. A recent study, however, showed that a $1 \mathrm{~kb}$ shorter Cas9 from Staphylococcus aureus (SaCas9) can perform genome editing with efficiency similar to that of SpCas9 [35].

In summary, the advance of new technologies is proceeding on multiple fronts. Together, these developments herald the advent of a new age.

\section{Disclosures}

The authors have nothing to declare.

\section{References}

1. Kim YG, Cha J, Chandrasegaran S (1996) Hybrid restriction enzymes: zinc finger fusions to Fok I cleavage domain. Proc Natl Acad Sci US A 93: 1156-1160.

2. Miller JC, Tan S, Qiao G, Barlow KA, Wang J, et al. (2011) A TALE nuclease architecture for efficient genome editing. Nat Biotechnol 29: 143-148.

3. Jinek M, Chylinski K, Fonfara I, Hauer M, Doudna JA, et al. (2012) A programmable dual-RNA-guided DNA endonuclease in adaptive bacterial immunity. Science 337: 816-821.
4. Ishino $Y$, Shinagawa H, Makino K, Amemura M, Nakata A (1987) Nucleotide sequence of the iap gene, responsible for alkaline phosphatase isozyme conversion in Escherichia coli, and identification of the gene product. J Bacteriol 169: 5429-5433.

5. Mojica FJ, Díez-Villaseñor C, Soria E, Juez G (2000) Biological significance of a family of regularly spaced repeats in the genomes of Archaea, Bacteria and mitochondria. Mol Microbiol 36: 244-246.

6. Mojica FJ, Díez-Villaseñor C, García-Martínez J, Soria 
E (2005) Intervening sequences of regularly spaced prokaryotic repeats derive from foreign genetic elements. $J$ Mol Evol 60: 174-182.

7. Bolotin A, Quinquis B, Sorokin A, Ehrlich SD (2005) Clustered regularly interspaced short palindrome repeats (CRISPRs) have spacers of extrachromosomal origin. Microbiology 151: 2551-2561.

8. Pourcel C, Salvignol G, Vergnaud G (2005) CRISPR elements in Yersinia pestis acquire new repeats by preferential uptake of bacteriophage DNA, and provide additional tools for evolutionary studies. Microbiology 151: 653-663.

9. Makarova KS, Grishin NV, Shabalina SA, Wolf YI, Koonin EV (2006) A putative RNA-interference-based immune system in prokaryotes: computational analysis of the predicted enzymatic machinery, functional analogies with eukaryotic RNAi, and hypothetical mechanisms of action. Biol Direct 1: 7 .

10. Barrangou R, Fremaux C, Deveau H, Richards M, Boyaval P, et al. (2007) CRISPR provides acquired resistance against viruses in prokaryotes. Science 315: 1709-1712.

11. Cong L, Ran FA, Cox D, Lin S, Barretto R, et al. (2013) Multiplex genome engineering using CRISPR/Cas systems. Science 339: 819-823.

12. Mali P, Yang L, Esvelt KM, Aach J, Guell M, et al. (2013) RNA-guided human genome engineering via Cas9. Science 339: 823-826.

13. Jinek M, East A, Cheng A, Lin S, Ma E, et al. (2013) RNA-programmed genome editing in human cells. Elife 2: e00471.

14. Cho SW, Kim S, Kim JM, Kim JS (2013) Targeted genome engineering in human cells with the Cas9 RNAguided endonuclease. Nat Biotechnol 31: 230-232.

15. Wang H, Yang H, Shivalila CS, Dawlaty MM, Cheng AW, et al. (2013) One-step generation of mice carrying mutations in multiple genes by CRISPR/Cas-mediated genome engineering. Cell 153: 910-918.

16. Horii T, Tamura D, Morita S, Kimura M, Hatada I (2013) Generation of an ICF syndrome model by efficient genome editing of human induced pluripotent stem cells using the CRISPR system. Int J Mol Sci 14: 19774-19781.

17. Horii T, Morita S, Kimura M, Kobayashi R, Tamura D, et al. (2013) Genome engineering of mammalian haploid embryonic stem cells using the Cas9/RNA system. PeerJ 1: e230.

18. Li HL, Gee P, Ishida K, Hotta A (2015) Efficient genomic correction methods in human iPS cells using CRISPR-Cas9 system. Methods [Epub ahead of print].

19. Xue W, Chen S, Yin H, Tammela T, Papagiannakopoulos T, et al. (2014) CRISPR-mediated direct mutation of cancer genes in the mouse liver. Nature 514: 380-384.

20. Yang L, Güell M, Niu D, George H, Lesha E, et al. (2015) Genome-wide inactivation of porcine endoge- nous retroviruses (PERVs). Science 350: 1101-1104.

21. Wu Y, Liang D, Wang Y, Bai M, Tang W, et al. (2013) Correction of a genetic disease in mouse via use of CRISPR-Cas9. Cell Stem Cell 13: 659-662.

22. Long C, McAnally JR, Shelton JM, Mireault AA, Bassel-Duby R, et al. (2014) Prevention of muscular dystrophy in mice by CRISPR/Cas9-mediated editing of germline DNA. Science 345: 1184-1188.

23. Yin H, Xue W, Chen S, Bogorad RL, Benedetti E, et al. (2014) Genome editing with Cas9 in adult mice corrects a disease mutation and phenotype. Nat Biotechnol 32: 551-553.

24. Holt N, Wang J, Kim K, Friedman G, Wang X, et al. (2010) Human hematopoietic stem/progenitor cells modified by zinc-finger nucleases targeted to CCR5 control HIV-1 in vivo. Nat Biotechnol 28: 839-847.

25. Perez-Pinera P, Kocak DD, Vockley CM, Adler AF, Kabadi AM, et al. (2013) RNA-guided gene activation by CRISPR-Cas9-based transcription factors. Nat Methods 10: 973-976.

26. Gilbert LA, Larson MH, Morsut L, Liu Z, Brar GA, et al. (2013) CRISPR-mediated modular RNA-guided regulation of transcription in eukaryotes. Cell 154: 442-451.

27. Chen B, Gilbert LA, Cimini BA, Schnitzbauer J, Zhang W, et al. (2013) Dynamic imaging of genomic loci in living human cells by an optimized CRISPR/Cas system. Cell 155: 1479-1491.

28. Fujita T, Fujii H (2013) Efficient isolation of specific genomic regions and identification of associated proteins by engineered DNA-binding molecule-mediated chromatin immunoprecipitation (enChIP) using CRISPR. Biochem Biophys Res Commun 439: 132-136.

29. Wang T, Wei JJ, Sabatini DM, Lander ES (2014) Genetic screens in human cells using the CRISPR-Cas9 system. Science 343: 80-84.

30. Chen S, Sanjana NE, Zheng K, Shalem O, Lee K, et al. (2015) Genome-wide CRISPR screen in a mouse model of tumor growth and metastasis. Cell 160: 1246-1260.

31. Findlay GM, Boyle EA, Hause RJ, Klein JC, Shendure J (2014) Saturation editing of genomic regions by multiplex homology-directed repair. Nature 513: 120-123.

32. Ran FA, Hsu PD, Lin CY, Gootenberg JS, Konermann S, et al. (2013) Double nicking by RNA-guided CRISPR Cas9 for enhanced genome editing specificity. Cell 154: 1380-1389.

33. Kleinstiver BP, Prew MS, Tsai SQ, Topkar VV, Nguyen NT, et al. (2015) Engineered CRISPR-Cas9 nucleases with altered PAM specificities. Nature 523: 481-485.

34. Zetsche B, Gootenberg JS, Abudayyeh OO, Slaymaker IM, Makarova KS, et al. (2015) Cpfl Is a Single RNAGuided Endonuclease of a Class 2 CRISPR-Cas System. Cell 163: 759-771.

35. Ran FA, Cong L, Yan WX, Scott DA, Gootenberg JS, et al. (2015) In vivo genome editing using Staphylococcus aureus Cas9. Nature 520: 186-191. 